\title{
Caracterización molecular de la región determinante de resistencia a quinolonas (QRDR) de la topoisomerasa IV de Bartonella bacilliformis en aislados clínicos
}

\section{Molecular characterization of quinolones resistance determining region (QRDR) of Bartonella bacilliformis topoisomerasa IV in clinical isolates}

\author{
Abraham Espinoza-Culupú1, Ruth Quispe-Gaspar', Michael Jaramillo', Melisa Icho', Anika Eca', Pablo \\ Ramírez ${ }^{1}$, Débora Alvarado', Juan Carlos Guerrero², Franklin Vargas-Vásquez ${ }^{3}$, Ofelia Córdova ${ }^{4}$, Ruth \\ García-de-la-Guarda ${ }^{1 *}$
}

1 Laboratorio de Microbiología Molecular y Biotecnología - Facultad de Ciencias Biológicas Universidad Nacional Mayor de San Marcos, Perú. Apartado postal 110058, Lima-11, Perú.

2 Establecimiento de Salud I-4 Huancabamba, Piura, Perú

3 Instituto de Investigación en Microbiología y Parasitología Tropical - Universidad Nacional de Trujillo, Perú

4 Laboratorio de Biología Celular y Molecular Dpto. de Ciencias - Universidad Privada Antenor Orrego, Trujillo, Perú.

*Autor para correspondencia R. García-de-laGuarda: rgarciad@unmsm.edu.pe

Email A. Espinoza: aespinozac20@gmail.com Email R. Quispe: ruty_lilly@yahoo.es Email M. Jaramillo: maycol776@yahoo.es Email M. Icho: m.biomicro@gmail.com Email A. Eca: anikaeca@gmail.com Email P. Ramírez: pramirezr@unmsm.edu.pe Email D. Alvarado: dalvaradoi@unmsm.edu.pe Email J.C. Guerrero: guerreroruizjc@hotmail.com Email F. Vargas-Vásquez: frvargasv@yahoo.es Email O. Córdova: omacop@hotmail.com

Citación:

Espinoza-Culupú A., R. Quispe-Gaspar, M. Jaramillo, M. Icho, A. Eca, P. Ramírez, D. Alvarado, J.C. Guerrero, F. Vargas-Vásquez, O. Córdova, R. García-de-la-Guarda. 2014. Molecular characterization of quinolones resistance determining region (QRDR) of Bartonella bacilliformis topoisomerasa IV in clinical isolates. Revista peruana de biología 21(1): 089-098 (Mayo 2014), doi: http:// doi.org/10.15381/rpb.v21i1.8251

\section{Resumen}

Bartonella bacilliformis es el agente etiológico de la Enfermedad de Carrión, endémica del Perú. Pocas investigaciones han sido realizadas acerca de los genes asociados a la resistencia antimicrobiana en aislados clínicos de este patógeno. Estos genes no están caracterizados molecularmente, ni se conoce la región asociada a dicha resistencia. Por ello, el objetivo del este trabajo fue caracterizar molecularmente la región determinante de la resistencia a las quinolonas (QRDR) en la topoisomerasa IV, que está codificada por los genes parC y parE, así como también desarrollar una prueba de susceptibilidad antimicrobiana para $B$. bacilliformis Las muestras sanguíneas de 65 pacientes procedentes de La Libertad, Cusco, Ancash y Piura, se sembraron en placas de agar sangre e incubaron a $30{ }^{\circ} \mathrm{C}$ con $5 \% \mathrm{CO}_{2}$. Luego se procedió a: (1) determinar la susceptibilidad antimicrobiana y (2) extraer el DNA genómico, amplificar los genes mencionados, secuenciarlos y analizarlos mediante herramientas bioinformáticas. Se obtuvieron 6 cultivos positivos. Los aislados fueron sensibles a la ciprofloxacina (excepto uno procedente de Quillabamba-Cusco, que presentó susceptibilidad disminuida) y resistentes al ácido nalidíxico. Del análisis de las secuencias aminoacídicas de ParC y ParE de $B$. bacilliformis se concluye que presentan diferencias aminoacídicas en comparación con las secuencias de las proteínas respectivas de E. coliK12 MG1655, que probablemente confieran resistencia al ácido nalidíxico pero no a la ciprofloxacina. Se determinó que las QRDR de las proteínas ParC y ParE de B. bacilliformis están comprendidas entre los aminoácidos 67 al 118 y 473 al 530 , respectivamente. El antibiograma y la concentración mínima inhibitoria se evalúan mejor usando inóculos a escala 1 de McFarland y a los 6 días de incubación.

Palabras clave: Susceptibilidad antimicrobiana; Bartonella bacilliformis; ParC; ParE; quinolonas.

\section{Abstract}

Bartonella bacilliformis is the etiologic agent of Carrion's disease, which if endemic to Peru. Studies on antimicrobial resistance genes from clinical isolates of this pathogen are scarce, and the molecular characteristics of these genes and their region resistance-associated are currently unknown. In this work we made the molecular characterization of the quinoloneresistance, and establish the region (QRDR) for the topoisomerase IV, which is encoded by the parC and parE genes, as well as develop an antimicrobial susceptibility test for $B$. bacilliformis. 65 Blood samples from La Libertad, Cusco, Ancash and Piura were processed on Blood Agar plates and incubated at $30{ }^{\circ} \mathrm{C}, 5 \% \mathrm{CO}_{2}$. The antimicrobial susceptibility was determined, then the genomic DNA extracted, aforementioned genes amplified, their sequence determined and it analyzed using bioinformatics tools. Six positive cultures were obtained. The isolates were susceptible to Ciprofloxacin (except one strain from Quillabamba - Cusco, which showed decreased susceptibility) and were resistant to Nalidixic Acid. From the sequence analysis of $B$. bacilliformis ParC and ParE there have been shown amino acid differences compared to the respective protein sequences from $E$. coli K12 MG1655, which is likely to confer resistance to Nalidixic Acid but not to Ciprofloxacin. It was determined that $B$. bacilliformis ParC and ParE proteins QRDRs are comprised between amino acids 67 to 118 and 473 to 530 , respectively. The antibiogram and the minimal inhibitory concentration are best assessed using the \#1 McFarland standards after a 6-day incubation period.

Keywords: antimicrobial susceptibility; Bartonella bacilliformis; ParC; ParE; quinolones.

$\begin{array}{ll}\text { Presentado: } & 18 / 01 / 2014 \\ \text { Aceptado: } & 30 / 04 / 2014 \\ \text { Publicado online: } & 26 / 05 / 2014\end{array}$

Journal home page: http://revistasinvestigacion.unmsm.edu.pe/index.php/rpb/index

(c) Los autores. Este artículo es publicado por la Revista Peruana de Biología de la Facultad de Ciencias Biológicas, Universidad Nacional Mayor de San Marcos. Este es un artículo de acceso abierto, distribuido bajo los términos de la Licencia Creative Commons Atribución-NoComercial-Compartirlgual 4.0 Internacional.(http://creativecommons.org/licenses/by-nc-sa/4.0/), que permite el uso no comercial, distribución y reproducción en cualquier medio, siempre que la obra original sea debidamente citadas. Para uso comercial, por favor póngase en contacto con editor.revperubiol@gmail.com. 


\section{Introducción}

Bartonella bacilliformis es el agente etiológico de la Enfermedad de Carrión, endémica de los valles interandinos del Perú. Esta enfermedad es de notificación obligatoria en el Perú y sus tasas de incidencia por regiones han variado a lo largo de décadas, con un número elevado de casos en Amazonas, Ancash y Piura en los últimos ańos (DGE, 2013). Se han usado diferentes antibióticos para el tratamiento de infecciones con $B$. bacilliformis, tales como eritromicina, cloranfenicol, ciprofloxacina, rifampicina, entre otros (MINSA 2006, Rolain et al. 2004). El tratamiento con estos antibióticos produce una rápida reducción de la bacteremia, sin embargo, se ha reportado casos en que la bacteremia persiste después de suspender el tratamiento con antibióticos (Rolain et al. 2004, Henriquez et al. 2004, Perez et al. 2010, Biswas 2010).

Actualmente, la ciprofloxacina (Cip) es considerada como el tratamiento de primera línea en Perú, debido a su buena penetración celular (MINSA 2006, Tarazona et al. 2006). Existen reportes donde se menciona que el $22.6 \%$ de los pacientes presentaron bacteremia asintomática crónica después del tratamiento con Cip (Pachas 2000), a pesar que este antibiótico aparentemente es exitoso (Maguińa et al. 2001, Maguińa et al 2008, Rolain et al. 2004). Además estudios previos han demostrado una susceptibilidad disminuida de $B$. bacilliformis a la Cip, también como a otras fluoroquinolonas (Flores 2008, Dörbecker et al. 2006, Sobraques et al. 1999). Asimismo, se ha determinado que las fluoroquinolonas usadas en monoterapias pueden ser inefectivas porque se han aislado fácilmente $B$. bacilliformis resistentes a Cip in vitro (Biswas et al. 2007). Recientemente, se ha determinado que $B$. bacilliformis presenta resistencia constitutiva a la quinolona ácido nalidíxico ( $\mathrm{Nal}$ ) (Del Valle et al. 2010). Asimismo, se ha demostrado que existen aislados de $B$. bacilliformis que están adquiriendo resistencia a rifampicina y eritromicina además de Cip (Minnick et al. 2003, Biswas et al. 2007). Maguińa et al. (2008), afirman que la mayoría de estos tratamientos se han definido basándose en opinión de expertos o estudios pequeños controlados, haciéndose necesaria la ejecución de ensayos clínicos randomizados controlados para la búsqueda de un mejor tratamiento.

Actualmente existen pocas investigaciones acerca de la susceptibilidad a antimicrobianos in vitro de $B$. bacilliformis. No existe un antibiograma estandarizado para esta bacteria y tampoco se conocen los mecanismos de resistencia ni las secuencias de los genes asociados a dicha resistencia. La base molecular de la resistencia a las quinolonas, como ocurre en otros microorganismos, reside en mutaciones en la región determinante de resistencia a quinolonas (QRDR) de los genes gyrA, gyrB de la topoisomerasa II y los genes $\operatorname{par} C$ y parE de la topoisomerasa IV (Law et al. 2010, Serra 2008, Hopkins et al. 2005).

Por consiguiente, el objetivo del presente estudio fue caracterizar la secuencia de la QRDR de ParC y ParE de aislados clínicos de $B$. bacilliformis de zonas endémicas, así como estandarizar un procedimiento para realizar el antibiograma y la concentración mínima inhibitoria (CMI).

\section{Material y métodos}

Pacientes y muestras.- Se colectaron 65 muestras de sangre venosa en tubos al vacío con anticoagulante EDTA, a personas con síntomas de la Enfermedad de Carrión (DGE 2013), según su historia clínica, para lo cual nos asistía el personal del centro de salud de la zona, con autorización verbal del Jefe de la Región de Salud respectiva dada después de haberle presentado el proyecto de la investigación.

Los muestreos fueron realizados en las siguientes zonas endémicas de la Enfermedad de Carrión (DGE 2013): a) Sondor $(\mathrm{n}=8)$, Sondorrillo $(\mathrm{n}=12)$ y Carmen de la Frontera $(\mathrm{n}=23)$, Departamento de Piura, b) Urubamba ( $n=5)$ y Quillabamba $(\mathrm{n}=10)$, Departamento de Cusco, c) Calipuy $(\mathrm{n}=4)$, Departamento de La Libertad, y d) Caraz $(\mathrm{n}=3)$, Departamento de Ancash.

Declaración de ética.- El estudio fue aprobado bajo los lineamientos del Comité de Ética de la Facultad de Ciencias Biológicas de la Universidad Nacional Mayor de San Marcos. Todos los pacientes o adultos responsables firmaban por escrito el consentimiento informado para la toma de muestra y su posterior análisis. Los pacientes fueron informados que la muestra de sangre sería utilizada para fines de investigación y tomaban la decisión si aceptaban o rechazaban la toma de muestra.

Aislamiento de $\boldsymbol{B}$. bacilliformis.- Para el aislamiento de esta bacteria a partir de sangre de pacientes, se emplearon placas de agar Columbia suplementado con $5 \%$ de glóbulos rojos de ovino y $2 \%$ de suero bovino fetal, así como tubos con medio bifásico compuestos por una fase sólida del mismo medio antes descrito y una fase líquida de caldo infusión cerebro corazón (BHI) (Colichón \& De Bedon 1973; Coleman \& Minnick 2001). Las placas se sembraron por diseminación con $200 \mu \mathrm{L}$ de sangre homogeneizada y los tubos con medio bifásico con la adición de 800 a $1000 \mu \mathrm{L}$ de sangre, siguiendo la metodología de Quispe (2009). Los cultivos se incubaron a $30{ }^{\circ} \mathrm{C}$ con $5 \%$ de $\mathrm{CO}_{2}$ a una humedad relativa de $100 \%$ (Quispe 2009; Minnick et al. 2003) por seis a ocho días hasta su crecimiento. Los subcultivos y los ensayos de susceptibilidad antimicrobiana se realizaron en agar Columbia base (Merck) suplementado con $8 \%$ de glóbulos rojos de ovino.

Determinación de la susceptibilidad antimicrobiana en Bartonella bacilliformis.- Se empleó la técnica de Kirby-Bauer (CLSI 2011, Restrepo 2002; Perilla et al. 2003) modificada según lo indicado por Pendle et al. (2006) para los ensayos de difusión en disco, y la prueba Épsilon para la CMI. Los cultivos de $B$. bacilliformis aislados de pacientes y las cepas del Instituto Pasteur de Francia CIP57.17 y CIP57.18, se resuspendieron en un volumen de $3 \mathrm{~mL}$ de solución salina fisiológica a $\mathrm{pH} 7.2$ a una concentración celular equivalente a las escalas $0.5,1$ y 2 de McFarland. Se inoculó un volumen de $1.5 \mathrm{~mL}$ de las suspensiones bacterianas en las placas, bañando toda la superficie y aspirando el exceso con una micropipeta. Luego se procedió a colocar los discos de antibiótico (Oxoid) e incubar a $30^{\circ} \mathrm{C}$ con $5 \%$ de $\mathrm{CO}_{2}$ y a una humedad relativa de $100 \%$. La evaluación del crecimiento e inhibición bacteriana en las placas se realizaron desde el día 5 hasta el 12. Para la susceptibilidad bacteriana se emplearon los siguientes antimicrobianos: discos de Cip (5 $\mu \mathrm{g})$ y de $\mathrm{Nal}(30 \mu \mathrm{g})$, y tiras de Cip con una gradiente de concentración de 0.02 a $32 \mu \mathrm{g} / \mu \mathrm{L}$ (tiras MICE de Oxoid).

Extracción de DNA.- La extracción del DNA genómico de las bartonelas, provenientes de cultivos en placas, se realizó usando el kit Miniprep de Wizard Genomic Purification (Promega), siguiendo las indicaciones del fabricante. 
Lavado del paquete celular.- Se hicieron 3 lavados de las células bacterianas con agua de grado molecular para eliminar los restos de medio de cultivo, centrifugando a $13000 \mathrm{rpm}$ por 2 minutos y descartando el sobrenadante.

Lisis celular y acción de la RNasa.- Al sedimento de células bacterianas se le adicionó $600 \mu \mathrm{l}$ de la solución de lisis e incubó a $80{ }^{\circ} \mathrm{C}$ por 5 minutos, luego se dejó a temperatura ambiente por 5 minutos para adicionarle $3 \mu \mathrm{L}$ de RNasa $(4 \mathrm{mg} / \mathrm{mL})$ y se incubó a $37^{\circ} \mathrm{C}$ por 1 hora.

Precipitación de proteínas.- Se agregó $200 \mu \mathrm{L}$ de solución de precipitación de proteínas al lisado tratado con RNasa, se homogeneizó vigorosamente por 20 segundos e incubó por 5 minutos en hielo. Luego se centrifugó a $13000 \mathrm{rpm}$ por $3 \mathrm{mi}-$ nutos colocando el sobrenadante a un tubo de microcentrifuga de $1.5 \mathrm{~mL}$.

Precipitación de DNA.- Al tubo de microcentrifuga con sobrenadante, se adicionó $600 \mu \mathrm{L}$ de isopropanol absoluto mezclando suavemente por inversión, hasta formar una masa visible de DNA, para luego centrifugar a $13000 \mathrm{rpm}$ por 2 minutos, eliminando el sobrenadante cuidadosamente y escurriendo el tubo en papel absorbente limpio.

Lavado del DNA.- Al microtubo que contenía el sedimento de DNA se adicionó $600 \mu \mathrm{L}$ de etanol al 70\%, se mezcló por inversión varias veces para lavar el sedimento, luego se centrifugó a $13000 \mathrm{rpm}$ por 2 minutos, se eliminó el sobrenadante cuidadosamente y se dejó secar el tubo de 10 a 15 minutos.

Hidratación del DNA.- El DNA fue rehidratado con 100 $\mu \mathrm{L}$ de solución de rehidratación de DNA por incubación a $65^{\circ} \mathrm{C}$ por 1 hora, periódicamente se golpeaba el tubo suavemente para mezclar la solución. Finalmente se guardó a $-20^{\circ} \mathrm{C}$ hasta su uso.

Ensayo de PCR.- La amplificación por PCR del gen ialB de B. bacilliformis se realizó para confirmar la especie (Mitchell \& Minnick 1995, Flores 2008). Posteriormente, se amplificó las regiones QRDR de los genes parCy parE de la topoisomerasa IV. Los iniciadores usados fueron ParC F: TCTTATGCTAAGTGTGCACGGA y ParC R: TACCAACAGCAATCCCTGAAGAA (Flores, 2008), ParE F: GAAGTCGCACGAGAGCGCAA y ParE R: AGCGGAACCGTTCTTCCGGT (diseńados en este estudio). El parámetro de ciclaje fue: desnaturalización a $95^{\circ} \mathrm{C}$ durante 3 minutos ( 1 ciclo), seguido de 29 ciclos a $94^{\circ} \mathrm{C}$ por 30 segundos, hibridación a $57^{\circ} \mathrm{C}(\operatorname{parC}), 58^{\circ} \mathrm{C}($ ialB $)$ y $62^{\circ} \mathrm{C}$ (parE) por 30 segundos, extensión a $72{ }^{\circ} \mathrm{C}$ por 30 segundos y extensión final a $72^{\circ} \mathrm{C}$ por 7 minutos. La mezcla de reacción en un volumen de $50 \mu \mathrm{L}$ fue: $1.5 \mu \mathrm{L}$ de cada iniciador $(10 \mu \mathrm{M}), 5$ $\mu \mathrm{L}$ de dNTPs $(2 \mathrm{mM}), 5 \mu \mathrm{L}$ de buffer Kod Hot Start (10X), 3 $\mu \mathrm{L}$ de $\mathrm{MgSO}_{4}(25 \mathrm{mM}), 1.5 \mu \mathrm{L}$ de Taq KOD Hot Start (Novagen), $31.5 \mu \mathrm{L}$ de agua grado molecular y $1 \mu \mathrm{L}$ de DNA molde.

Para visualizar el DNA y los productos de amplificación, se hicieron electroforesis en gel de agarosa al $1 \%$ en buffer TAE $0,5 \mathrm{X}$, mezclando las muestras con solución de carga $6 \mathrm{X}$ (Novagen ${ }^{\circ}$. Se utilizó el marcador de tamaño molecular Perfect DNA $^{\mathrm{TM}} 50$ bp Ladder (Novagen ${ }^{\circ}$. Luego de la electroforesis, los geles se tiñeron con bromuro de etidio $(0.5 \mu \mathrm{g} / \mathrm{mL})$, por 45 segundos, para visualizarlos en el transiluminador UV.

Secuenciamiento y análisis bioinformático.- Los amplificados fueron secuenciados por la empresa Macrogen, con secuenciamiento estándar y por ambas direcciones. El análisis in silico se realizó mediante herramientas bioinformáticas empleando Bioedit (Hall 1999) y MEGA 5.2 (Tamura et al. 2011) para evaluar y obtener la secuencia consenso de los genes. Posteriormente se usó CLUSTALW 2.1 (Thompson et al. 1994) para los alineamientos múltiples y determinar las posibles mutaciones en la secuencias nucleotídicas y aminoacídicas, comparando nuestras secuencias con las depositadas en la base de datos GenBank para los genes parC y parE de B. bacilliformis KC583 y E. coli K-12 MG1655.

Modelamiento por homología del dominio QRDR de ParC y ParE de B. bacilliformis relacionadas a la resistencia antimicrobiana.- Con las secuencias aminoacídicas de $B$. bacilliformis se realizaron los modelamientos para localizar, en la estructura, los aminoácidos involucrados en la resistencia antimicrobiana. Para estos modelamientos se empleó el servidor online SWISS-MODEL (http://swissmodel.expasy.org/). Los modelos fueron obtenidos en formato pdb y las estructuras tridimensionales se visualizaron con Swiss-Pdb Viewer 4.1.0 y Jmol13.

Obtención de las secuencias de genes relacionados a resistencia antimicrobiana en el GenBank.- Se accedió al GenBank para obtener las secuencias de los genes de la topoisomerasa IV de B. bacilliformis KC583 parC y parE (Gene ID: 4684170 y 4684565$)$, y para $E$. coli cepa K-12 MG1655 (Gene ID: 947499 y 947501) para compararlas con nuestras secuencias obtenidas.

Finalmente las secuencias nucleotídicas de los aislados de $B$. bacilliformis, obtenidos en el presente estudio, fueron ingresadas a la base de datos EBI (http://www.ebi.ac.uk/) con los números de acceso siguientes: HG315965, HG315966, HG315967 para el gen parC y HG315962, HG315963, HG315964 para el gen parE

\section{Resultados}

De los muestreos realizados se obtuvieron seis cultivos positivos $(9 \%)$ con características culturales compatibles con B. bacilliformis. A los cultivos positivos se les codificó con USM-LMMB (Universidad Nacional Mayor de San Marcos - Laboratorio de Microbiología Molecular y Biotecnología), seguido del número del aislado: USM-LMMB-001 (Calipuy - La Libertad); USMLMMB-002 y USM-LMMB-006 (Quillabamba - Cuzco); USM-LMMB-003 (Urubamba- Cuzco); USM-LMMB-005 (Sondor- Piura); USM-LMMB-007 (Carmen de la frontera - Piura). Los seis cultivos fueron positivos a la amplificación del gen $i a l B$, indicando que los cultivos corresponden a $B$. bacilliformis.

Susceptibilidad antimicrobiana en Bartonella bacilliformis.- Se ha estandarizado el procedimiento para realizar el antibiograma y la CIM (mediante la prueba Épsilon) para determinar la susceptibilidad antimicrobiana de $B$. bacilliformis. Se obtuvieron crecimientos uniformes en las placas, siendo el inóculo con el que se visualizaron mejor los halos de inhibición del crecimiento bacteriano, el que estaba a una concentración celular equivalente al tubo $\mathrm{N}^{\circ} 1$ de la escala de McFarland (Fig. 1, Tabla 1, Fig. 2 y Tabla 2). En cuanto al tiempo de incubación óptimo para hacer la medición del diámetro de dichos halos, éste resultó ser el día 6 después de la siembra de las placas, debido a que fue el menor tiempo en el que se observaron los halos mejor definidos (Tablas 1 y 2). 


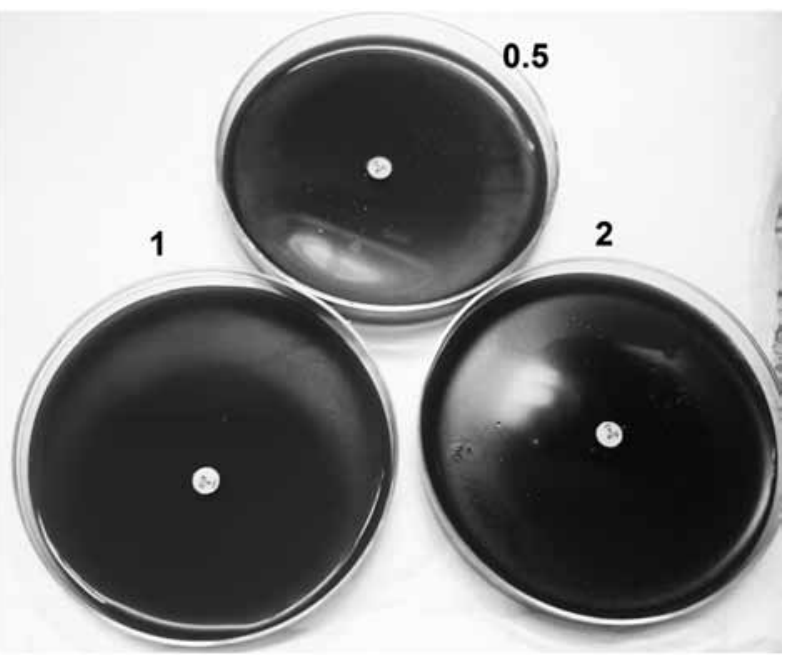

Figura 1.- Estandarización del procedimiento para realizar el antibiograma mediante difusión en agar con disco de Cip $(5 \mu \mathrm{g})$ para determinar la susceptibilidad antimicrobiana de $B$. bacilliformis. Los ensayos se hicieron con la cepa USMLMMB-005, ajustando los inóculos a los tubos $\mathrm{N}^{\circ} 0.5$ (placa superior), 1 (placa de la izquierda) y 2 (placa de la derecha) de la escala de McFarland. Las lecturas se hicieron hasta los 12 días.

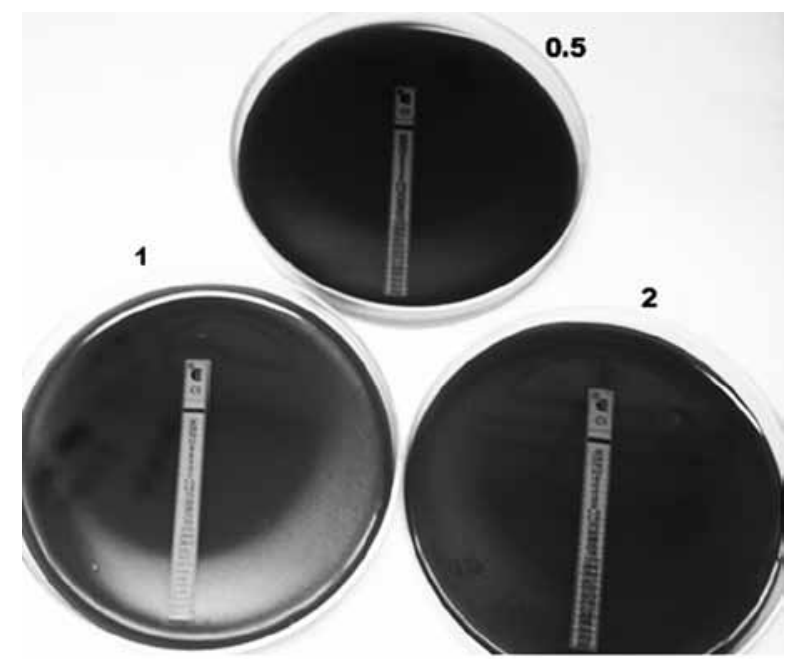

Figura 2.- Estandarización del procedimiento para realizar la CIM mediante la prueba Épsilon con tiras de Cip con una gradiente de concentración de 0.02 a $32 \mu \mathrm{g} / \mu \mathrm{L}$ para determinar la susceptibilidad antimicrobiana de $B$. bacilliformis. Los ensayos se hicieron con la cepa USM-LMMB-005, ajustando los inóculos a los tubos $\mathrm{N}^{\circ} 0.5$ (placa superior), 1 (placa de la izquierda) y 2 (placa de la derecha) de la escala de McFarland. Las lecturas se hicieron hasta los 12 días.
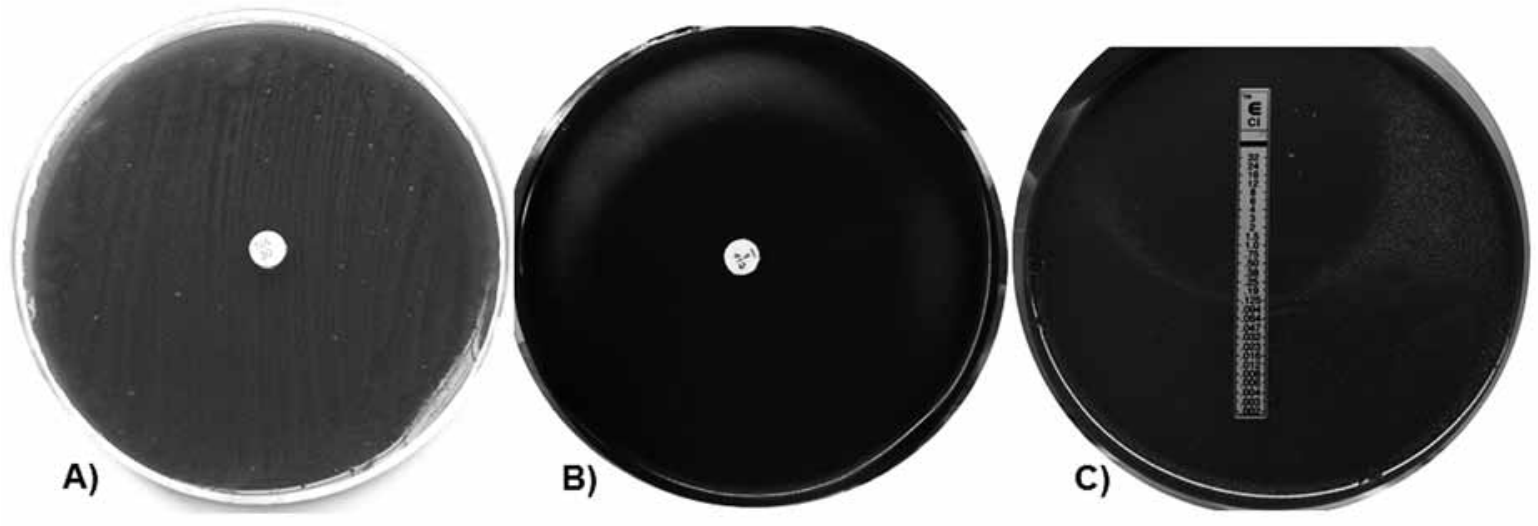

Figura 3.- Susceptibilidad antimicrobiana de B. bacilliformis USM-LMMB-005: En A, mediante disco difusión en agar para el ácido nalidixico $(6 \mathrm{~mm}$, resistente) y en B, para la Cip $(52 \mathrm{~mm}$, sensible). En C, prueba Épsilon para Cip (0.023 mg/L). Los ensayos se hicieron usando una suspensión celular equivalente a la escala 1 de McFarland, evaluándose hasta los 12 dias de incubación.

Tabla 1.- Valores del diámetro en milímetros de los halos de inhibición del crecimiento bacteriano de $B$. bacilliformis en la prueba de susceptibilidad antimicrobiana a la Cip, mediante difusión por disco en agar, con inóculos ajustados a tres tubos de la escala de McFarland y mediciones de los halos en diferentes tiempos de incubación.

\begin{tabular}{ccccc}
\hline & \multicolumn{4}{c}{ Tiempo de incubación (días) } \\
\cline { 2 - 5 } Escala McFarland & 5 & 6 & 7 & 12 \\
\hline 0,5 & N.D & $52(E D)$ & 52 & 52 \\
1 & $52(E D)$ & 52 & 52 & 52 \\
2 & $52(E D)$ & 52 & 52 & 52 \\
\hline
\end{tabular}

ND: No definido.

ED: Empezando a definirse el halo.
Tabla 2.- Valores para $B$. bacilliformis de la CIM en $\mathrm{mg} / \mathrm{L}$ mediante la prueba Épsilon con tiras de Cip, con inóculos ajustados a tres tubos de la escala de McFarland y evaluaciones en diferentes tiempos de incubación.

\begin{tabular}{ccccc}
\hline & \multicolumn{4}{c}{ Tiempo de incubación (días) } \\
\cline { 2 - 5 } Escala McFarland & 5 & 6 & 7 & 12 \\
\hline 0,5 & N.D & $0.023(E D)$ & 0.023 & 0.023 \\
1 & $0.023(E D)$ & 0.023 & 0.023 & 0.023 \\
2 & $0.032(E D)$ & 0.032 & 0.032 & 0.032 \\
\hline
\end{tabular}

ND: No definido.

ED: Empezando a definirse el halo. 


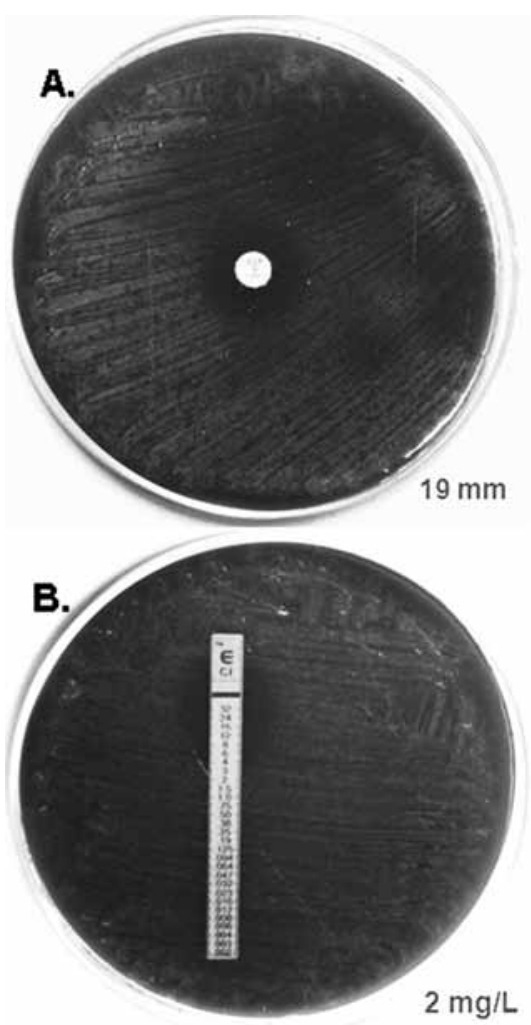

Figura 4. Susceptibilidad disminuida a la ciprofloxacina de la cepa Bartonella bacilliformis USM-LMM-002: A. Prueba Épsilon que determinó una CMI de $2 \mathrm{mg} / \mathrm{L}$ con tiras de Cip (gradiente de concentración de 0.02 a $32 \mu \mathrm{g} / \mu \mathrm{L}$ ), B. Halo de inhibición del crecimiento de $19 \mathrm{~mm}$ con disco de Cip $(5 \mu \mathrm{g})$.

Con respecto a la susceptibilidad de los aislados obtenidos, todos mostraron resistencia al Nal (Fig. 3A), y fueron sensibles a Cip (Fig. 3B y 3C), a excepción del aislado USM-LMM-002 procedente de Quillabamba - Cusco, que mostró susceptibilidad disminuida a Cip (Fig. 4).

Amplificación de genes de la topoisomerasa IV de Bartonella bacilliformis. - Las amplificaciones de las QRDR de los genes de la topoisomerasa IV se evidenciaron mediante electroforesis en gel de agarosa al 1\%. Los carriles del 1 al 4 corresponden al amplificado de la QRDR del gen parC con un tamaño de 349 pb, y los carriles del 5 al 8 al amplificado de la QRDR del gen parE con un tamaño de $1495 \mathrm{pb}$ como se muestra en la Fig. 5.

Análisis in silico de la subunidad A (ParC) y subunidad B (ParE) de la topoisomerasa IV de Bartonella bacilliformis.- $\mathrm{El}$ análisis se hizo comparando las secuencias de ParC y ParE de $B$. bacilliformis con las de $E$. coli $\mathrm{K} 12$ debido a que en esta bacteria se conocen mejor las QRDR y las sustituciones de aminoácidos en las posiciones que alteran la susceptibilidad a las quinolonas. De esta manera hemos deducido las posiciones equivalentes en B. bacilliformis.

Secuencias aminoacídicas de ParC.- En el análisis de las secuencias aminoacídicas de ParC de los aislados de B. bacilliformis, se encontró que existen diferencias a nivel de los aminoácidos 80 y 94 (Ser por Ala) dentro de la QRDR, comparando con la QRDR de ParC de E. coli K12. Además se encontraron otros cambios muy cercanos al sitio activo en los aminoácidos 123 y 129 también de Ser por Ala. El aislado USM-LMMB-003

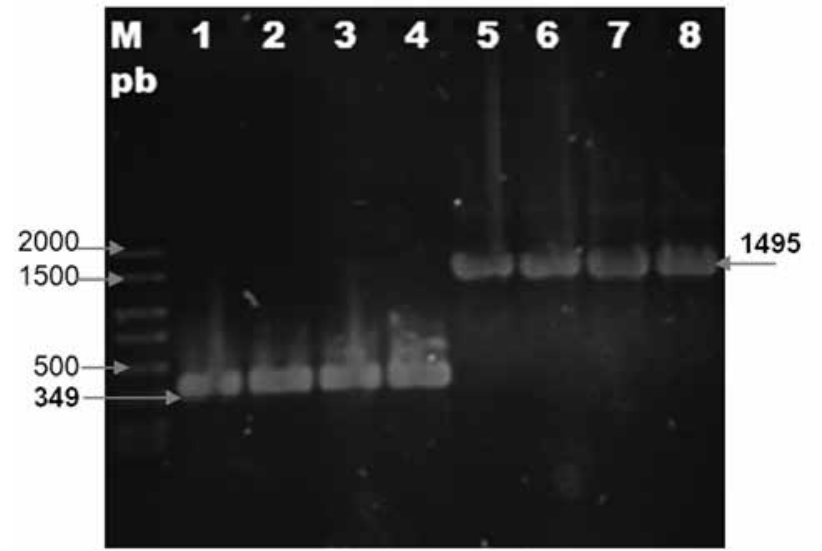

Figura 5.- Amplificados de la QRDR de los genes de la topoisomerasa IV: gen parC con tamaño de 349 pb correspondientes a los carriles del 1 al 4 y gen parE con tamaño de 1495 pb correspondiente a los carriles del 5 al 8 . Se utilizaron las siguientes cepas: CIP57.17, carriles 1 y 5 ; USM-LMMB-005, carriles 2 y 6 ; USM-LMMB-006 carriles 3 y 7 ; USM-LMMB-007, carriles 4 y 8 . M1 corresponde al marcador Perfect DNA ${ }^{\mathrm{TM}} 50$ bp Ladder Novagen ${ }^{\circledR}$.

presentó diferencias aminoacídicas (Tyr por Asp) fuera de la QRDR (color celeste, Fig. 6) en los aminoácidos 124, 149, 164, 167 y 175 (numeración correspondiente a B. bacilliformis) incluso en el sitio activo.

Secuencias aminoacídicas de ParE.- En el análisis de las secuencias aminoacídicas de ParE, no se encontró ninguna diferencia dentro de la QRDR entre los aislados, pero sí se encontraron diferencias en relación a $E$. coli $\mathrm{K} 12$, en el aminoácido 441 (Lys por Arg), que es el más reportado para cepas con resistencia a fluoroquinolonas en E. coli. También en el aminoácido 451 se puede observar un cambio de Ser por Ala como se muestra en la Fig. 7B. Analizando las secuencias nucleotídicas del gen parE de los aislados de B. bacilliformis, se encontraron en USM-LMMB005 dos mutaciones puntuales a nivel nucleotídico, las cuales no produjeron cambios en los aminoácidos 328 y 458 (Fig. 7A).

Modelamiento por homología del dominio QRDR de ParC y ParE de B. bacilliformis relacionadas a la resistencia antimicrobiana.- En la estructura terciaria del QRDR de ParC y ParE de $B$. bacilliformis se pudo apreciar cambios en la estructura terciaria en las posiciones donde existe una diferencia aminoacídica de Ser por Ala, cuando se le compara con $E$. coli K12. Estos cambios sugieren una débil interacción con la quinolona, los cambio son resaltados en círculo rojo como se observan en la Figuras 8 y 9

\section{Discusión}

La Enfermedad de Carrión aún continúa siendo un problema de salud pública en Perú, a pesar de su importancia actualmente existen pocos estudios sobre la resistencia antimicrobiana de este patógeno. El presente estudio es a nuestro entender el primer trabajo sobre caracterización molecular de genes asociados a la resistencia antimicrobiana de aislados de $B$. bacilliformis de zonas endémicas del Perú. Este trabajo es de gran importancia, porque a pesar que las especies de Bartonella son altamente susceptibles a pruebas de antibióticos (Dörbecker et al. 2006), existen reportes 
QRDR

B. b-USM-LMMB-005

B.b-RC583

B.b-USM-LMMB-001

B.b-USM-LMMB-002

B.b-USM-LMMB-003

B.b-USM-LMMB-006

B.b-USM-LMMB-007

B.b-CIP57.17

E.c-str.K-12-MG1655

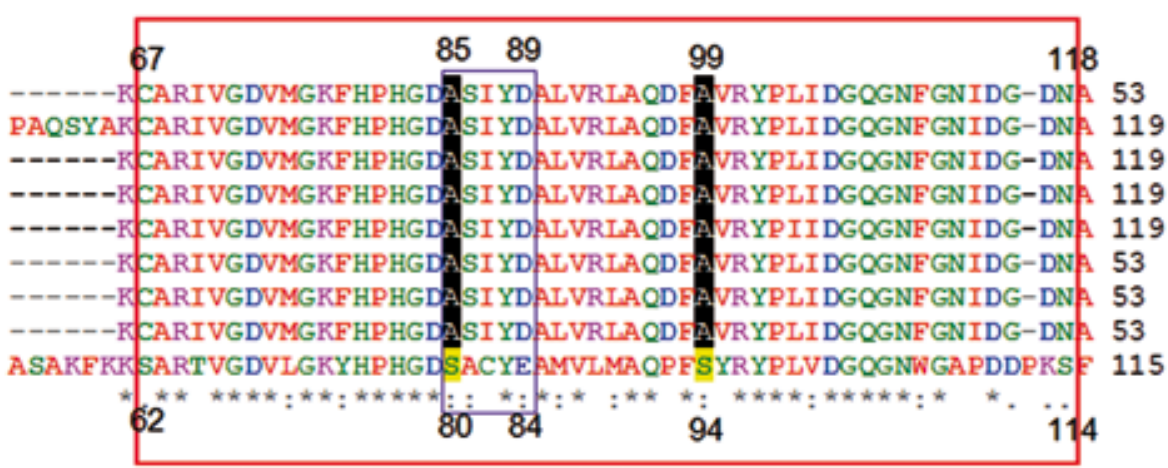

149

164167

175

AAMRYTE RRMTEVELLEGINENAIDFRL̈TYNEEDEEPIVLPGÄFPN̄LLANGSSG̈---- 109 AAMRYTEARMTEV PELLLEGINENAIDFRLTYNEEDEEPIVLPGAFPNLLANGSSGIAVG 179

A.AMRYTEPRMTEVPELLLEGINENAIDFRLTYNEEDEEPIVLPGAFPNLLANGSSGIAVG 179 AAMRYTENRMTEV PELLLEGINENAIDFRLTYNEEDEEPIVLPGAFPNLLANGSSGIAVG 179 AAMRDTEARMTEV ELLLEGINENAIDFR TYNEEDEEPIVLPG FP FLLANGSS IAVG 179 AAMRYTEARMTEVBELLLEGINENAIDFRLTYNEEDEEPIVLPGAFPNLLANGSSG---- 109 AAMRYTEARMTEV PELLLEGINENAIDFRLTYNEEDEEPIVLPGAFPNLLANGSSG---- 109 AAMRYTERRMTEVBELLLEGINENAIDFRLTYNEEDEEPIVLPGAFPNLLANGSSG---- 109 AAMRYTESRLSKYSELLLSELGQGTADWVPNFDGTLOEPKMLPARLPNILLNGTTGIAVG 175

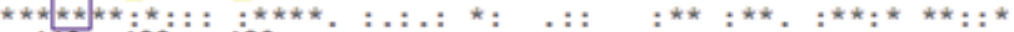

11993129

activo

Figura 6.- Alineamiento múltiple de las secuencias aminoacídicas de ParC de la cepa de Bartonella bacilliformis KC 583 y de las aisladas, comparadas con ParC de E. coli K12, utilizando el programa ClustalW 2.1. Las sustituciones aminoacídicas (Ser por Ala) están resaltadas en color negro. La numeración en la parte inferior corresponde a las posiciones de los aminoácidos de la proteína ParC de E. coli (NP_417491.1) y en la parte superior su equivalente en B. bacilliformis (YP_989154.1).

\section{A}

B.b-CIP57.17

B. b-RC583

B.b-USM-IMMB-006

B.b-USM-LMMB-005
B.b-USM-LMMB-007
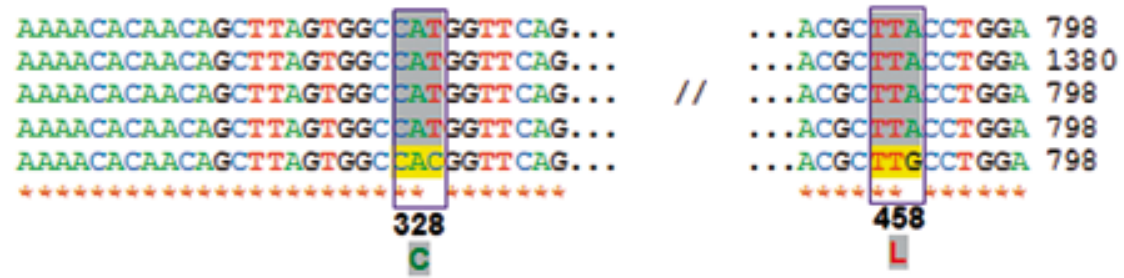

QRDR

\section{B}

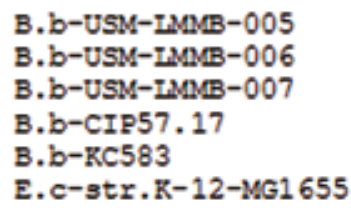

B.b-USM-IMOM-005

B.b-CIP57.17

E.c-str. R-12-NG1655

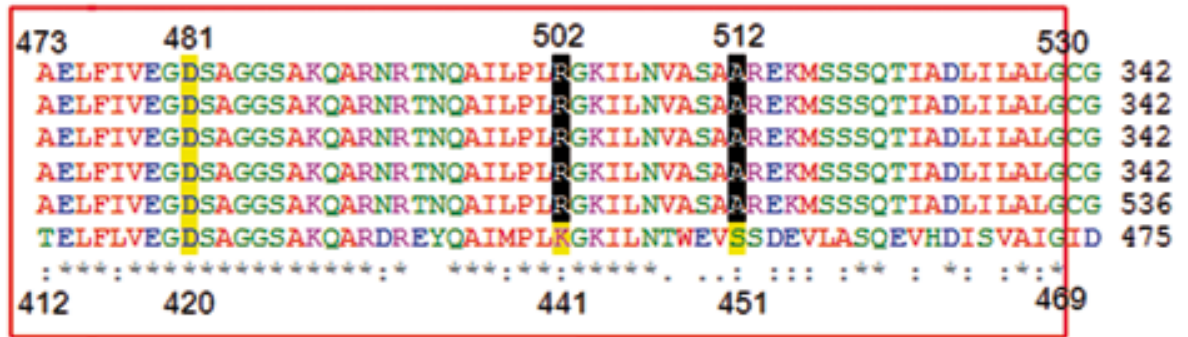

Figura 7.- Alineamiento múltiple de secuencias nucleotídicas de los aislados de Bartonella bacilliformis y de la cepa KC583. En A, el alineamiento muestra las mutaciones puntuales del aislado USM-LMMB-005 que no produjeron cambios en el aminoácido respectivo y en $\mathrm{B}$, el alineamiento muestra las secuencias aminoacídicas de ParE de la cepa de Bartonella bacilliformis KC583 y las aisladas, comparadas con ParE de E. coli K12, utilizando el programa ClustalW 2.1. Se indica sus respectivos aminoácidos cambiantes (Lys por Arg) resaltado en color negro. La numeración en la parte inferior corresponde a las posiciones de los aminoácidos de la proteína ParE de E. coli (NP_417502.1) y en la parte superior su equivalente para $B$. bacilliformis (YP_989184.1). 
A)

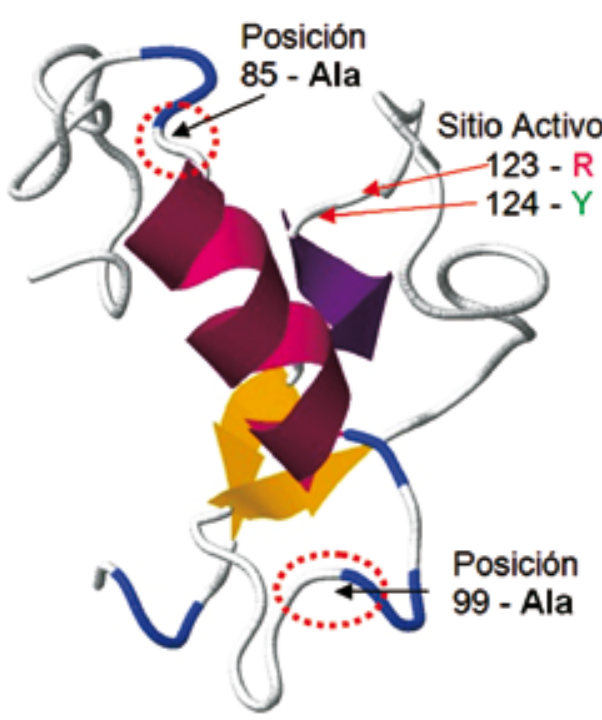

B)

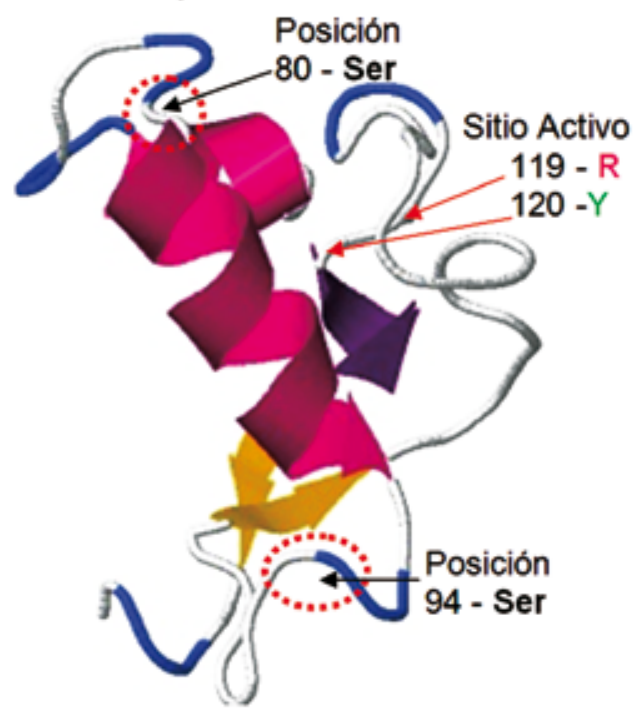

Figura 8.-. Estructura tridimensional de la QRDR de ParC de Bartonella bacilliformis por homología con ParC de Escherichia coli K12 (Protein Data Base (PDB): 1ZVU). En A, se muestra la QRDR de B. bacilliformis y en B, la QRDR de E. coli K12 MG1655. Las flechas de color negro indican la posición donde existen diferencias aminoacídicas entre $B$. bacilliformis y $E$. coli $\mathrm{K} 12$, observando un cambio en la estructura para $B$. bacilliformis, que se resalta en un círculo rojo. Las flechas rojas indican las posiciones correspondientes al sitio activo.

A)

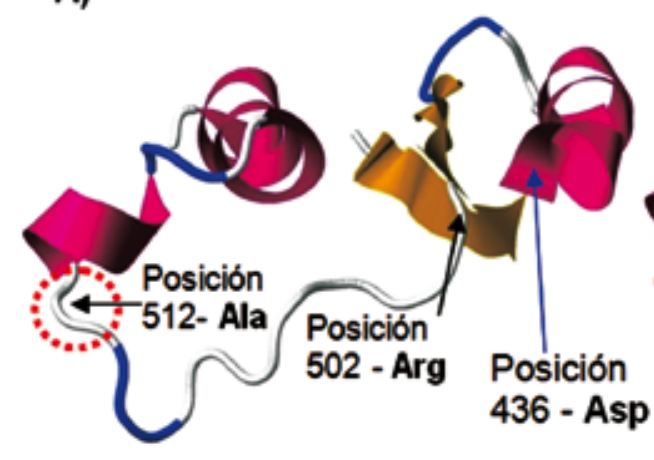

B)

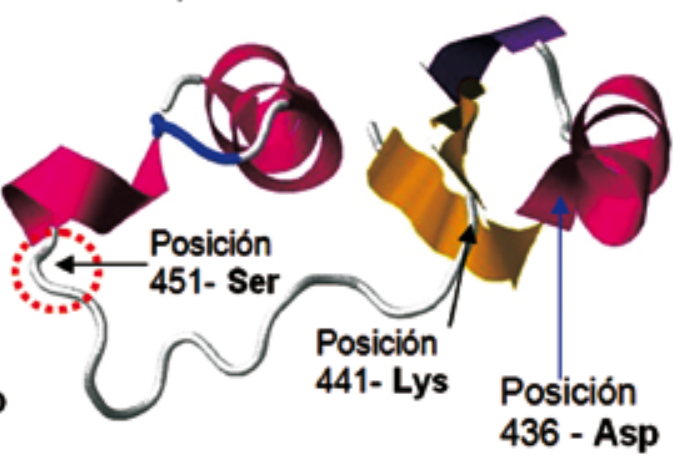

Figura 9.- Estructura tridimensional de la QRDR de ParE de Bartonella bacilliformis por homología con ParE de Acinetobacter baumannii (PDB: 2XKK). En A, se muestra la región de la QRDR de $B$. bacilliformis y en B, la QRDR de E. coli K12 MG1655. Las flechas de color negro indican la posición donde existen diferencias aminoacídicas entre $B$. bacilliformis y $E$. coli $\mathrm{K} 12$, observando un cambio en la estructura para $B$. bacilliformis, que se resalta en un círculo rojo. La flecha azul indica la localización del aminoácido Asp, que es importante porque se han reportado cepas resistentes a quinolonas cuando hay mutación en esta posición.

Tabla 3.- Valores obtenidos de la susceptibilidad antimicrobiana de las cepas de Bartonella bacilliformis ensayadas para ciprofloxacina y ácido nalidíxico.

\begin{tabular}{lccccc}
\hline & E- & & \multicolumn{2}{c}{ KirbyBauer $(\mathrm{mm})$} \\
\cline { 2 - 2 } \cline { 5 - 6 } CEPAS & Cip & & Cip & AN \\
\hline CIP 57.17 & 0.125 & & 46 & 6 \\
CIP 57.18 & 0.015 & & 55 & 6 \\
USM-LMMB-001 & 0.125 & & 50 & 6 \\
USM-LMMB-002 & 2 & & 19 & 6 \\
USM-LMMB-003 & 0.125 & & 50 & 6 \\
USM-LMMB-005 & 0.023 & & 52 & 6 \\
USM-LMMB-006 & 0.023 & & 50 & 6 \\
USM-LMMB-007 & 0.19 & & 78 & 6 \\
\hline
\end{tabular}


de fallas en la monoterapia para las enfermedades relacionadas a Bartonella usando beta-lactámicos, macrólidos, tetraciclina, rifampicina o fluoroquinolonas (Biswas et al. 2007). Los aislados de $B$. bacilliformis presentaron una CIM para Cip que oscilaba entre 0.19 y $0.023 \mathrm{mg} / \mathrm{L}$ (Tabla 3), la que concuerda con los valores de CIM descritos en la literatura (Sobraquès et al. 1999, Döbecker et al. 2006, Angelakis et al. 2008). En cuanto a la susceptibilidad mediante Kirby-Bauer, los aislados analizados mostraron resistencia al $\mathrm{Nal}$ (primera quinolona) y sensibilidad a la Cip (Fig. 3).

El análisis in silico de las secuencias aminoacídicas de ParC y ParE de B. bacilliformis se hizo comparando las secuencias de éstas en $E$. coli K12 debido a que en esta bacteria se conocen mejor las QRDR y las sustituciones de aminoácidos que alteran la susceptibilidad a las quinolonas. Mediante esta comparación hemos deducido las posiciones equivalentes de los aminoácidos entre E. coli y B. bacilliformis tal como lo realizaron Del Valle et al. (2010).

De acuerdo con nuestros resultados, la QRDR de la proteína ParC (subunidad A de la topoisomerasa IV) de Bartonella bacilliformis comprendería desde el aminoácido 67 al 118 (Fig. 7). El análisis de la QRDR de ParC revela que todos los aislados de $B$. bacilliformis presentan sustituciones de Ser por Ala en los aminoácidos 85 y 99 (numeración equivalente a 80 y 94 en E. coli) en comparación a ParC de E. coli K12 (Fig. 6). Las mutaciones en los aminoácidos 78, 80 y 84 de la QRDR de ParC de E. coli K12, son las más descritas y relacionadas a la resistencia a quinolonas (Hopkins et al. 2005, Serra 2008). En las posiciones 119 (Arg) y $120(\mathrm{Tyr})$ que corresponden al sitio activo de esta enzima, no se encontraron sustituciones aminoacídicas. Además, las posiciones 123 y 129 cercanas al sitio activo, también presentan cambios de Ser por Ala. Estas sustituciones dentro de la QRDR también han sido encontradas en Bartonella spp. y otras bacterias intracelulares como Tropheryma whipplei en la que se ha reportado heterogeneidad de la susceptibilidad frente a las fluoroquinolonas (Masselot et al. 2003, Angelakis et al. 2008, Angelakis et al. 2009). Varios estudios mencionan que las mutaciones de Ser por Ala en dichas posiciones están asociadas a la resistencia natural a las fluoroquinolonas (Cambau et al. 1994, Rodríguez et al. 2001, Del Valle et al. 2010). Estos estudios explicarían la resistencia al $\mathrm{Nal}$ en todos los aislados de B. bacilliformis obtenidos en el presente trabajo. Es importante señalar que a pesar de encontrar las mutaciones para la resistencia a la quinolona $\mathrm{Nal}$, posiblemente otras mutaciones en el gen $\operatorname{par} C$ de estas bacterias o en otros genes deberían ser necesarias para que ocurra la resistencia a las fluoroquinolonas (como por ejemplo a la Cip). Actualmente, la Cip parece ser efectiva en el tratamiento recomendado por el MINSA, sin embargo existe adquisición de resistencia por mutaciones acumuladas en estos genes. Por tanto, como fue recomendado en otros estudios (Angelakis et al. 2008, Del Valle et al. 2010) se debe de evitar el uso de Cip como monoterapia en el tratamiento de esta enfermedad.

Los resultados de este estudio sugieren que la QRDR de ParE (subunidad B de la DNA topoisomerasa IV) de B. bacilliformis está comprendida entre los aminoácidos 473 y 530 , como se muestra en la Fig. 7. La mayoría de mutaciones descritas en la QRDR de ParE de E. coli K12, que es homóloga es GyrB (Ruiz et al. 1987), están entre los aminoácidos 412 y 469. El análisis de las secuencias aminoacídicas de ParE de B. bacilliformis no reveló cambios entre las secuencias, sin embargo, dentro de la QRDR se presentaron dos sustituciones aminoacídicas en las posiciones 441 (Lys por Arg) y 451 (Ser por Ala) en comparación con E. coli K12. La posición 420, resaltada en amarillo (Fig. 7), se encontró sin cambios. Los cambios tanto en la posición 420 como 441 y sus equivalentes en $B$. bacilliformis (481 y 502, respectivamente), son las más citadas en cepas resistentes a quinolonas en E.coli (Hopkins et al. 2005, Sorlozano et al. 2007, Jiménez et al. 2009). También se han reportado cambios en los aminoácidos 416 (Leu por Phe), 445 (Leu por His) y 458 (Ser por Ala). Estas mutaciones ya descritas (Hopkins et al. 2005, Sorlozano et al. 2007, Jiménez et al. 2009) no fueron encontradas en las secuencias de $B$. bacilliformis del presente estudio. Las mutaciones en otras posiciones podrían ser importantes para la resistencia o disminución de la susceptibilidad, como lo es el cambio en el aminoácido 451, que en E coli K12 es una Ser y su equivalente en $B$. bacilliformis es Ala. Las sustituciones del mismo tipo (Ser por Ala) reportadas en el aminoácido 458, confieren resistencia en cepas de $E$. coli productoras de $\beta$ lactamasas de espectro extendido (Sorlozano et al. 2007). De los aislados de $B$. bacilliformis, USM-LMMB-005 presentó mutaciones puntuales en el nucleótido de la tercera posición, las que no produjeron cambios en el aminoácido respectivo (328 y 458). El aminoácido 458 se encuentra cercano a la QRDR de ParE, por lo que es probable que los cambios nucleotídicos adicionales a éstos puedan contribuir a generar resistencia. Justamente este aislado, USM-LMMB-005 procedente de la localidad de Sondorillo es donde se reportaron fallas al tratamiento con Cip. Es posible que en las cepas de B. bacilliformis circulantes en esta localidad se acumulen mutaciones en las secuencias de estos genes asociados a la resistencia a Cip, lo que podría estar contribuyendo a la resistencia para este grupo de antibióticos; es por ello que en esta localidad se trata a los pacientes con la Enfermedad de Carrión, con ceftriaxona en vez de Cip.

Mediante herramientas bioinformáticas se realizó el modelamiento de la probable estructura tridimensional de la QRDR de las proteínas ParC y ParE de B. bacilliformis (Fig. 8 y 9). Para esto, se realizó una comparación con la estructura de la topoisomerasa IV de Escherichia coli y A. baumannii, mostrando las sustituciones que existen en relación con E. coli K12, sin embargo, al evaluar la estructuras terciarias de las QRDR se apreciaron cambios estructurales colocando un círculo punteado de color rojo en éstos, sugiriendo una débil interacción de la quinolona y su blanco, los cambios observados de Ser por Al, podrían estar alterando los patrones de hidrofobicidad que se relacionan con la estructura de la proteína.

En conclusión, se obtuvieron seis aislados de B. bacilliformis, todos fueron resistentes al $\mathrm{Nal}$, sensibles a Cip y uno de ellos, USM-LMMB-002 procedente de Quillabamba - Cusco, presentó susceptibilidad disminuida a la Cip. Se determinó que las QRDR de las proteínas ParC y ParE de B. bacilliformis están comprendidas entre los aminoácidos 67 al 118 y 473 al 530, respectivamente. Se sugiere que el cambio con relación a $E$. coli K12 de Ser por Ala en ParC de B. bacilliformis determinaría la resistencia al $\mathrm{Nal}$ pero no a la Cip. Con respecto al antibiograma y a la CMI (con tiras de la prueba Épsilon) para B. bacilliformis, concluimos que los halos de inhibición del crecimiento bacteriano se visualizan mejor cuando las placas Petri se siembran por "inundación" bañándolas con inóculos de $1.5 \mathrm{~mL}$ a la escala 1 de McFarland, e incubándolas durante 6 días. 


\section{Agradecimientos}

Al Consejo Nacional de Ciencia y Tecnología (CONCYTEC) por el financiamiento al proyecto $\mathrm{N}^{\circ} 323-2010-\mathrm{CONCYTEC-}$ OAJ y al VRI-UNMSM Proyecto $\mathrm{N}^{\circ} 111001032$. A los técnicos de laboratorio Nexar Alvarado, Jesús Paico y Rosa Pintado del Centro de Salud de Huancabamba - Piura. Al Dr. Jorge Cortez y a Teófanes Paredes del Centro de Salud de Calipuy - Stgo. de Chuco, La Libertad; al Dr. Manuel Montoya del Cusco; al Biólogo Paul Pacheco Román del Centro de Salud de UrubambaCusco; a la Técnica de laboratorio Flor Céspedes del Centro de Salud de Uchumayo - Quillabamba, Cusco; y a los biólogos José Luis Ramos Coveñas y Martín Nizama Teixeira; todos ellos por brindarnos su apoyo en la toma de muestras. A los Dres. Joaquim Ruiz del Hospital Clinic de Barcelona y Luis Del Valle de la Universitat Politècnica de Catalunya, por su apoyo en la revisión del manuscrito de la tesis de Ruth Quispe, algunos de cuyos resultados están incluidos en el presente artículo. Esta publicación es parte de la tesis para optar al grado de Magíster en Biología Molecular de Abraham Espinoza Culupú, bajo la asesoría de Ruth García de la Guarda.

\section{Literatura citada}

Angelakis E., S. Biswas, C. Taylor, et al. 2008. Heterogeneity of susceptibility to fluoroquinolones in Bartonella isolates from Australia reveals a natural mutation in gyrA. J Antimicrob Chemother. 61(6):1252-1225. doi: 10.1093/jac/dkn094

Angelakis E., D. Raoult\&J.M. Rolain. 2009. Molecular characterization of resistance to fluoroquinolones in Bartonella henselae and Bartonella quintana. J Antimicrob Chemother. 63(6):1288-1289. doi: 10.1093/jac/dkp133.

Biswas S., D. Raoult, \& J.M. Rolain. 2007. Molecular mechanisms of resistance to antibiotics in Bartonella bacilliformis. J Antimicrob Chemother. 59(6):1065-1070. doi: 10.1093/ jac/dkm105

Biswas S. \& J.M. Rolain. 2010. Bartonella infection: treatment and drug resistance. Future Microbiol. 5(11):1719-1731. doi:10.2217/fmb.10.133.

Cambau E., W. Sougakoff, M. Besson, et al. 1994. Selection of a gyrA mutant of Mycobacterium tuberculosis resistant to fluoroquinolones during treatment with ofloxacin. J Infect Dis. 170(5):1351.

CLSI (Clinical and Laboratory Standards Institute). 2011. Performance Standards for Antimicrobial Susceptibility Testing; TwentyFirst Informational Supplement.M100-S21 Vol. $31 \mathrm{~N}^{\circ} .1$. Wayne, Pensylvania, USA.

Coleman S.A. \& M.F. Minnick. 2003. Differential expression of the invasion-associated locus B (ialB) gene of Bartonella bacilliformis in response to environmental cues. Microb Pathog. 34(4):179-186. http://dx.doi.org/10.1016/S08824010(03)00005-6

Colichon H. \& C.F. De Bedon. 1973. Carrion's disease: useful nutrients for the growth of Bartonella bacilliformis. Rev Latinoam Microbiol. 15(2):75-79.

Del Valle L.J., L. Flores, M. Vargas, R. Garcia-de-la-Guarda, et al. 2010. Bartonella bacilliformis, endemic pathogen of the Andean region, is intrinsically resistant to quinolones. Int J Infect Dis. 14(6):506-510. doi:10.1016/j.ijid.2009.07.025

DGE (Dirección General de Epideniología). 2013. Sala situacional para el Análisis de Situación de Salud. Enfermedad de Carrión $<$ http://www.dge.gob.pe/portal/index.php?option=com_co ntent\&view=article\&id=14\&Itemid=154>

Dörbecker C., A. Sander, K. Oberle, et al. 2006. In vitro susceptibility of Bartonella species to 17 antimicrobial compounds: comparison of E-test and agar dilution. J Antimicrob Chemother.58(4):784-788. doi: 10.1093/jac/dkl341

Flores L. 2008.Estudios moleculares en Bartonella bacilliformis para el control de la Enfermedad de Carrión. Tesis para optar el grado académico de Magíster en Biología Molecular. Facultad de Ciencias Biológicas, Universidad Nacional Mayor de San Marcos. 156 pp.
Hall T.A.1999. BioEdit: a user-friendly biological sequence alignment editor and analysis. <http://www.mbio.ncsu.edu/BioEdit/ bioedit.html >. [accedido 18/02/13].

Henriquez C., C. Hinojosa \& P. Ventosilla. 2004. Report of an unusual case of persistent bacteremia by Bartonella bacilliformis in a splenectomized patient. The American Society of Tropical Medicine and Hygiene 71(1): 53-55.

Hiroshi Y. \& R. Katsumata. 2006. Antibiotic resistance in bacteria and its future for novel antibiotic development. Journal of Bioscience Biotechnology and Biochemistry. 70(5): 10601075. http://dx.doi.org/10.1271/bbb.70.1060

Hopkins K.L., R.H. Davies \& E.J. Threlfall. 2005. Mechanisms of quinolone resistance in Escherichia coli and Salmonella: recent developments. Int J Antimicrob Agents. 25(5):358373. doi:10.1016/j.ijantimicag.2005.02.006

Jiménez A., A. Jimenez, M. Arrabal, et al. 2009. Resistencias a quinolonas en aislados clínicos de Escherichia coliproductores de betalactamasas de espectroextendido. Higiene y Sanidad Ambiental. 9: 449-466.

Law DK, M Shuel, S Bekal, et al. 2010. Genetic detection of quinolone resistance in Haemophilus parainfluenzae: Mutations in the quinolone resistance-determining regions of gyrA and parC. Canadian Journal of Infectious Diseases \& Medical Microbiology. 21:e20-2.

Maguiña C., P.J. Garcia, E. Gotuzzo, et al. 2001. Bartonellosis (Carrion's disease) in the modern era. Clinical Infectious Diseases 33(6): 772-779. doi: 10.1086/322614

Maguiña C., C. Ugarte-Gil, P. Breña, et al. 2008. Actualización de la Enfermedad de Carrión. Update of Carrion's disease. Rev Med Hered.19 (1): 036-041.

Masselot F., A. Boulos, M. Maurin, et al. 2003. Molecular evaluation of antibiotic susceptibility: Tropheryma whipplei paradigm. Antimicrobial Agents and Chemotherapy 47(5):1658-1664. doi: 10.1128/AAC.47.5.1658-1664.2003

Minnick M.F., Z.R. Wilson, L.S. Smitherman, et al. 2003. gyrA mutations in ciprofloxacin-resistant Bartonella bacilliformis strains obtained in vitro. Antimicrobial Agents and Chemotherapy 47(1): 383-386. doi: 10.1128/AAC.47.1.383386.2003

MINSA. 2006. Norma Técnica No 048-MINSA/DGSP-V.01. "Atención de la Bartonelosis o Enfermedad de Carrión en el Perú".74 pp.

Mitchell S.J \&M.F Minnick. 1995. Characterization of a two-gene locus from Bartonella bacilliformis associated with the ability to invade human erythrocytes. Infection and Immunity 63(4):1552-62.

Pachas P. 2000. Epidemiología de la Bartonelosis en el Perú.Módulos Técnicos - Serie documentos monográficos.Lima, PE: Oficina General de Epidemiología/InstitutoNacional de Salud. 83 pp.

Pendle S., A. Ginn \& J. Iredell. 2006. Antimicrobial susceptibility of Bartonella henselae using Etest methodology. Antimicrobial Agents and Chemotherapy 57(4):761-3. doi:10.1093/jacl dki485

Perez-Martinez L., J.R. Blanco \& J.A. Oteo. 2010. Treatment of human infections caused by Bartonella spp. Revista española de quimioterapia 23(3):109-114.

Perilla M., G. Ajello, Ch. Bopp, et al. 2003. Manual for the Laboratory Identification and Antimicrobial Susceptibility Testing of Bacterial Pathogens of Public Health Importance in the Developing World. CDC and WHO.186 pp.

Quispe R. 2009. Caracterización molecular de los genes asociados a la resistencia antimicrobiana en Bartonella bacilliformis. Tesis de Pre-Grado. Facultad de Ciencias Biológicas. E.A.P. Microbiología y Parasitología -Universidad Nacional Mayor de San Marcos. 65 pp.

Restrepo M., M. Robledo\& E. Bedoya. 2002. "Enfermedades Infecciosas", $5^{\mathrm{Ed}}$, Editorial Corporaciones para las Investigaciones Biológicas. Medellín-Colombia. 506 pp.

Rodríguez J.C., M. Ruíz, A. Climent, et al. 2001. In vitro activity of four fluoroquinolones against Mycobacterium tuberculosis. International Journal of Antimicrobial Agents 17(3):229231. doi.org/10.1016/S0924-8579(00)00337-X 
Rolain J.M., P. Brouqui, J.E. Koehler, et al. 2004. Recommendations for treatment of human infections caused by Bartonella species. Antimicrobial Agents and Chemotherapy 48(6):19211933. doi: 10.1128/AAC.48.6.1921-1933.2004

Ruiz J., S. Casellas, M.T. Jimenez de Anta, et al. 1997. The region of the parE gene, homologous to the quinolone-resistant determining region of the gyrB gene, is not linked with the acquisition of quinolone resistance in Escherichia coli clinical isolates. Antimicrobial Agents and Chemotherapy 39(6):839-840.

Serra A. 2008. Quinolonas. Facultad de Medicina UBA. Separata 2008- Vol $16 \mathrm{~N}^{\circ} 3$.

Sobraques M., M. Maurin, R.J. Birtles, et al. 1999. In vitro susceptibilities of four Bartonella bacilliformis strains to 30 antibiotic compounds. Antimicrobial Agents and Chemotherapy 43(8):2090-2092.
Sorlozano A., J. Gutierrez, A. Jimenez, et al. 2007. Contribution of a new mutation in parEto quinolone resistance in extendedspectrum-beta-lactamase-producing Escherichia coli isolates. Journal of Clinical Microbiology 45(8):2740-2742. doi: 10.1128/JCM.01093-07

Tarazona A., C. Maguińa, D. López de Guimaraes, et al. 2006. Terapia antibiótica para el Manejo de la Bartonelosis o Enfermedad de Carrión en el Perú. Rev Perú MedExp Salud Pública 23(3): 188-200.

Tamura K., D. Peterson, N. Peterson, et al. 2011.MEGA5: molecular evolutionary genetics analysis using maximum likelihood, evolutionary distance, and maximum parsimony methods. Molecular Biology and Evolution 28(10):2731-2739. doi: $10.1093 / \mathrm{molbev} / \mathrm{msr} 121$.

Thompson J., D.G. Higgins \& T.J. Gibson. 1994. CLUSTAL W: improving the sensitivity of progressive multiple sequence alignment through sequence weighting, position-specific gap penalties and weight matrix choice. Nucleic Acids Research 22(22):4673-80. doi:10.1093/nar/22.22.4673. 